\title{
An insight into new strategies to combat antifungal drug resistance
}

This article was published in the following Dove Press journal:

Drug Design, Development and Therapy

\author{
Yan-Hua Zheng' \\ Yue-Yun $\mathrm{Ma}^{2}$ \\ Yi Ding 3 \\ Xie-Qun Chen' \\ Guang-Xun Gao'
}

'Department of Hematology, Xijing Hospital, Fourth Military Medical

University, Xi'an, Shaanxi, China; ${ }^{2}$ Department of Clinical Laboratory Medicine, Xijing Hospital, Fourth Military Medical University, Xi'an, Shaanxi, China; ${ }^{3}$ Department of Pharmacy, Xijing Hospital, Fourth Military Medical University, Xi'an, Shaanxi, China
Correspondence: Guang-Xun Gao; Xie-Qun Chen

Department of Hematology, Xijing Hospital, Fourth Military Medical University, No 17 Chang'le West Road,

Xi'an 710032, Shaanxi, China

$\mathrm{Tel}+862984775199 ;+862984771857$

Fax +86 2984775205

Email gaoguangxun@fmmu.edu.cn; xiequnchen@fmmu.edu.cn

\begin{abstract}
Invasive fungal infections especially in immunocompromised patients represent a dominating cause of mortality. The most commonly used antifungal agents can be divided into three broad categories, including triazoles, echinocandins and polyenes. Antifungal resistance is on the increase, posing a growing threat to the stewardship of immunocompromised patients with fungal infections. The paucity of currently available antifungals leads to the rapid emergence of drug resistance and thus aggravates the refractoriness of invasive fungal infections. Therefore, deep exploration into mechanisms of drug resistance and search for new antifungal targets are required. This review highlights the therapeutic strategies targeting Hsp90, calcineurin, trehalose biosynthesis and sphingolipids biosynthesis, in an attempt to provide clinical evidence for overcoming drug resistance and to form the rationale for combination therapy of conventional antifungals and agents with novel mechanisms of action. What's more, this review also gives a concise introduction of three new-fashioned antifungals, including carboxymethyl chitosan, silver nanoparticles and chromogranin A-N46.
\end{abstract}

Keywords: carboxymethyl chitosan, chromogranin A-N46, Hsp90, calcineurin, trehalose, sphingolipids

\section{Introduction}

Fungal pathogens cause life-threatening infections in immunocompromised individuals, including those undergoing high-dose chemotherapy or radiotherapy for malignant cancer, those receiving immunosuppressive agents for autoimmune disease and for solid organ or hematopoietic stem cell transplantation and those infected with AIDS. ${ }^{1}$ Fungi are generally opportunistic pathogens, three most prevalent of which are Candida albicans, Cryptococcus neoformans and Aspergillus fumigatus. ${ }^{2}$ Therapeutic options for invasive fungal infections are restricted to three major structurally distinct classes of compounds, which are polyenes, triazoles and echinocandins. Most antifungal drugs function well by targeting sterol ergosterol of fungal cell membrane or the cell wall linker molecule $(1,3)-\beta$-D-glucan, or by hindering their biosynthesis. ${ }^{3}$

Azoles are the most widely used antifungals, which include fluconazole, voriconazole and posaconazole. Azoles function by inhibiting lanosterol demethylase, an enzyme implicated in the biosynthesis of ergosterol. Lanosterol demethylase is encoded by ERG11 in C. albicans and C. neoformans, and by CYP51A and CYP51B in A. fumigatus. Azoles also cause the accumulation of a toxic sterol intermediate referred to as $14 \alpha-$ methylergosta-8,24(28)-dien-6 $\beta, 3 \alpha$-diol, which is generated by Erg3. All these result in the depletion of ergosterol, destroying the integrity of fungal cell membranes. ${ }^{4}$

Echinocandins include caspofungin, micafungin and anidulafungin. Echinocandins bring into full play their fungicidal effect against Candida spp. and fungistatic effect 
against Aspergillus spp. by inhibiting $(1,3)-\beta$-D-glucan synthase, which is encoded by FKS1 in C. albicans and A. fumigatus, and by FKS1 and FKS2 in Candida glabrata and Saccharomyces cerevisiae. $(1,3)-\beta$-D-glucan, a structural polysaccharide, is a key component of the fungal cell wall, and inhibition of its synthesis leads to the impairment of the fungal cell wall. However, echinocandins have no potency to fight against Cryptococcus spp. ${ }^{5}$

Polyenes include amphotericin B (AmB) and nystatin, having a wide spectrum of activity against species of Candida, Cryptococcus and Aspergillus. In addition to the effect of inhibiting membrane transport proteins, amphipathic molecules also adhere to sterol ergosterol on the fungal cell membrane, forming drug-lipid complexes that intercalate into the fungal cell membrane and then drill pores in the cell membrane. Leakage of intracellular ions will destroy the proton gradient, thus resulting in osmotic cellular lysis. ${ }^{6,7}$

Currently, the limited therapeutic options for invasive fungal infections lead to antifungal resistance to a large extent. In addition, antifungal exposure under the circumstances of prophylactic use and repetitious or long-term treatment is closely correlated with the increasing occurrence of resistance, thus aggravating patient morbidity and mortality. What's more, mechanisms which confer antifungal resistance consist of biofilm formation, filamentation, genomic alterations and overexpression of drug efflux pumps (including major facilitator superfamily of transporters and ATP-binding cassette transporters). ${ }^{3,6}$ With the emergence of resistance to commonly used antifungal agents among opportunistic pathogens, it is imperative to find more effective approaches to eliminate this alarming threat. A better understanding of mechanisms of drug resistance contributes to searching for new targets and developing new antifungal therapies. This review focuses on some potential targets to overcome antifungal drug resistance.

\section{Targeting heat shock proteins}

Heat shock proteins (Hsps) act as molecular chaperones, which are responsible for folding and maintaining the proper conformation of client proteins. Hsp90 is a highly conserved molecular chaperone in eukaryotes that modulates the stability and activation of diverse client proteins, most of which play an important role in regulating cellular signal transduction. ${ }^{8}$ In fungi, Hsp90 exerts profound influences on cellular stress response circuitry via its interplay with a multitude of cellular regulators. ${ }^{9}$ Hsp90 consists of three functional domains. An N-terminal domain includes an ATP-binding pocket. A middle domain stabilizes client proteins and facilitates ATP binding and ATP hydrolysis, through which energy is provided for Hsp90 functioning. A C-terminal domain contains another nucleotide-binding site and regulates Hsp90 dimerization. ${ }^{10,11}$ The function of Hsp90 can be regulated by a variety of mechanisms, including ATP binding and hydrolysis, interactions with other co-chaperones and posttranslational modifications such as phosphorylation, methylation, acetylation and so on. ${ }^{12}$

As discussed above, azoles exert fungistatic activity by inhibiting lanosterol demethylase, thus hindering the synthesis of ergosterol. Meanwhile, accumulation of a toxic sterol intermediate generated by Erg3 can lead to severe cell membrane stress. Echinocandins cause disruption of cell wall integrity and cell wall stress by inhibiting $(1,3)$ $\beta$-D-glucan synthase. ${ }^{13}$ Incompetence of Hsp90 function also abrogates azole or echinocandin resistance induced by a wide range of mutations, including resistance that has already evolved in human hosts treated with azoles or echinocandin. ${ }^{14,15}$ Tolerance of two laboratory C. albicans strains (SC5314 and SN95) to micafungin was abrogated by pharmacological inhibition of Hsp90 with geldanamycin and by genetic compromise of their Hsp90 expression via substituting native promoter with the tetO promoter. ${ }^{14}$ Pharmacological inhibition of Hsp90 with geldanamycin reduces caspofungin resistance of an A. fumigatus clinical isolate. ${ }^{16} \mathrm{Hsp} 90$ inhibition also reduces resistance to caspofungin of C. glabrata clinical isolates. ${ }^{15}$ The combined use of Hsp90 inhibitors and echinocandins has fungicidal effect against azole-resistant strains of $A$. fumigatus. ${ }^{17}$ Inhibition of Hsp90 with geldanamycin significantly retards growth and proliferation of all clinical isolates when exposed to fluconazole, suggesting that azole resistance is abrogated by inhibiting Hsp90. ${ }^{18}$

\section{Upstream regulators}

Interactions with co-chaperones and posttranslational modifications serve as upstream regulators to govern drug resistance, altering Hsp90 conformation and thus influencing client protein recognition and chaperone function. Shapiro et al have established that Sgt1 functions as an Hsp90 co-chaperone in C. albicans, interacting physically with Hsp90 and potentiating basal tolerance and resistance to azoles and echinocandins. Meanwhile, depletion of Sgt1 also abrogates basal tolerance and acquired resistance to these two antifungals, activates PKA signaling, inhibits calcineurin activation and reduces virulence by inducing C. albicans filamentation. ${ }^{19}$ In C. albicans, stability and function of Hsp90 client proteins can be destroyed by deletion 
of regulatory subunit of protein kinase CK2 complex, thus crippling azole resistance. ${ }^{20}$

Inhibition of lysine deacetylases (KDACs) with trichostatin A destroys the stability and function of a myriad of Hsp90 client proteins, and abrogates resistance to azoles in C. albicans and $S$. cerevisiae. The crucial KDACs for drug resistance in $S$. cerevisiae are $\mathrm{Hda} 1$ and $\mathrm{Rpd} 3$, while KDACs in C. albicans remain to be an enigma. Despite the conservation of Hsp90, there exists wide divergence of KDACs between fungi and human beings, thus rendering targeting KDACs a promising strategy to specifically inhibit fungi without host toxicity. ${ }^{21}$

\section{Downstream effectors}

Hsp90 modulates drug resistance and virulence through various downstream effectors or client proteins such as calcineurin, the catalytic subunit of which interacts physically with Hsp90 in C. albicans. Hsp90 stabilizes the catalytic subunit of calcineurin, so depletion of Hsp90 leads to a decrease in calcineurin. ${ }^{14}$ Cyclosporin A and tacrolimus, the widely used immunosuppressants in clinics, fall into the category of calcineurin inhibitor. To develop calcineurin inhibitor analogs that retain antifungal effect without immunosuppressive activity in human beings holds great promise for abrogating drug resistance. ${ }^{17}$ Meanwhile, Hsp90 regulates resistance to both azoles and echinocandins via Mkc1 pathway. Depletion of Hsp90 results in disruption of $\mathrm{Mkc1}$, thus blockading downstream responses which potentiate fungi to survive the stress induced by antifungals. ${ }^{18}$

\section{Biofilm formation}

It is well established that biofilm is the predominant form of fungal growth in nature. Biofilm formation is another important element complicating antifungal therapy. A biofilm is a structured fungal community which is attached to the surface of some object and encapsulated within self-generated extracellular matrix, shielding pathogenic fungi against antifungals. A wide variety of biomaterials used in clinics, especially intravascular catheters, facilitate fungal colonization and biofilm formation. Candida spp. are the most frequently detected fungi on biomaterials. ${ }^{22}$ In C. albicans, inhibition of Hsp90 function in vitro not only decreases biofilm formation but also abrogates azole resistance, further retarding the biofilm cell dissemination which might serve as a source for newly found infection. In A. fumigatus, Hsp90 hindrance also reduces echinocandin resistance of biofilms in vitro. In a rat model with central venous catheter biofilm infection, azole used as a single agent has been found to be ineffective while powerful efficacy with little host toxicity was achieved after combination treatment with azoles involving genetic or pharmacological compromise of Hsp90 function. A good case in point is that after the biofilm was formed, the rat catheters were sterilized by co-administration of 17-allylamino-17-demethoxygeldanamycin, for pharmacological inhibition of Hsp90, and fluconazole. ${ }^{23}$

\section{Filamentation}

Hsp90 exerts a profound influence on the capacity of transition between the yeast form and the filamentous pseudohyphal or hyphal form in C. albicans. ${ }^{24}$ It is acknowledged that filaments play critical role in tissue invasion, expression of virulence factors such as adhesins and evasion from macrophages, all of which lead to deep-seated infections, while yeasts are implicated in early infection and disseminated infection. ${ }^{25}$ Compromise of Hsp90 function induces yeast-to-filament transition under various circumstances including serum exposure, nutrients deficiency, increased carbon dioxide and high temperature through novel circuitry composed of cyclin-dependent kinase Pho85, cyclin Pcl1 and transcriptional regulator $\mathrm{Hms} 1 .^{26}$

\section{Other Hsps}

Mayer et al have demonstrated that Hsp21 potentiates tolerance and resistance to commonly used antifungal drugs in C. albicans and have confirmed that Hsp21 functions in the same pathway as Hsp90. Compromise of Hsp21 leads to moderately enhanced susceptibility to AmB. An Hsp21defective mutant was incapable of proliferating in the presence of caspofungin, suggesting that anti-Hsp21 agents could be used in combination with other conventional antifungals. Meanwhile, deletion of Hsp21 renders C. albicans partially susceptible to tacrolimus. Hsp21 is also found in other fungal pathogens such as Candida dubliniensis, Candida tropicalis and Candida parapsilosis, but is not found in human beings. Above all, anti-Hsp21 drugs will meet the requirements of a novel anticandidal compound, destroying several kinds of pathogenic fungal cells with few side effects to patients. ${ }^{27,28}$ Recently, Hsp70, another molecular chaperone, has been revealed to play a key role in orchestrating stress response to caspofungin through Hsp90 and its co-chaperone StiA in A. fumigatus. Pharmacological inhibition of Hsp70 with pifithrin- $\mu$ or genetic deletion of StiA leads to the impairment of Hsp90 function and increased susceptibility to caspofungin, suggesting that targeting Hsp70-StiA-Hsp90 complex or inhibiting Hsp70 will enhance the antifungal efficacy against caspofungin-resistant A. fumigatus. ${ }^{29}$ 


\section{Targeting calcineurin}

Calcineurin, a highly conserved $\mathrm{Ca}^{2+}$-calmodulin (CaM)activated protein phosphatase $2 \mathrm{~B}$, takes important part in calcium-dependent signaling in various fungi. It consists of a catalytic subunit $(\mathrm{CnA})$ and a regulatory subunit $(\mathrm{CnB}){ }^{30}$ It is a heterodimer with $\mathrm{CnA}$ containing an $\mathrm{N}$-terminal phosphatase domain and $\mathrm{CnB}$-binding helix along with the CaM-binding domain. There exists an auto-inhibitory domain which represses the catalytic activity at the $\mathrm{C}$-terminus. The activity of calcineurin phosphatase is enhanced by adherence of $\mathrm{Ca}^{2+}-\mathrm{CaM}$ to $\mathrm{CnA}$ and $\mathrm{CnB}$, forming the activated complex $(\mathrm{CnA}-\mathrm{CnB}-\mathrm{CaM})$ through which $\mathrm{Ca}^{2+}$ signals are transmitted so as to dephosphorylate the transcriptional factor Crz1 and facilitate translocation of Crz1 into nucleus. Crz1, a fungal homolog of mammalian NFAT, functions by activating the transcription of genes which are involved in growth, virulence, cell wall integrity and drug resistance. Calcineurin activity can be inhibited by its binding to the immunophilinimmunosuppressant complexes, such as FK506-FKBP12 in tacrolimus and CsA-CypA in cyclosporine A. ${ }^{31}$

Calcineurin is crucial to growth, virulence, drug resistance, stress response and transition between morphological states for a broad spectrum of fungi, including C. albicans, A. fumigatus, C. neoformans and so on ${ }^{32,33}$ Combination of tacrolimus and caspofungin also has a synergistic effect against $C$. dubliniensis strain with echinocandin resistance. ${ }^{34}$ In C. albicans, combination of fluconazole and tacrolimus can partially reverse azole resistance. ${ }^{35}$ Combination of cyclosporin A with other antifungals also demonstrates synergistic effect against other Candida spp. ${ }^{36}$ Efficacy can be significantly enhanced by the combined application of calcineurin inhibitor and other antifungals in vitro or in experimental models of mucormycosis caused by Mucor and Rhizopus spp. ${ }^{37-40}$

It appears a powerful antifungal strategy to target the downstream effectors or substrates in the calcineurin signaling pathway via genetic and biochemical modifications. Crz1 regulates the transcriptional response induced by calcineurin activation in response to stress. Cbp1, an element which is responsible for hyphal growth, calcium homeostasis and regulation of calcineurin signaling, has been identified in C. neoformans and A. fumigatus. ${ }^{41}$

\section{Targeting trehalose biosynthetic pathway}

Trehalose, also referred to as $\alpha$-D-glucopyranosyl-(1,1)- $\alpha$ D-glucopyranoside, is a simple nonreducing disaccharide containing two glucose subunits connected by an $\alpha, \alpha-1,1-$ glycosidic linkage. Trehalose pathway is directly associated with glycolysis. Production of several important enzymes that play a pivotal role in trehalose formation, including trehalose-6-phosphate synthase (Tps1) and trehalose-6phosphate phosphatase (Tps2), is also regulated by trehalose pathway. ${ }^{42,43}$

Trehalose can be produced by bacteria, fungi, plants and invertebrates, while it cannot be created by mammalian hosts since there is no enzyme machinery for trehalose pathway in mammals. Blockade of trehalose pathway via inhibiting its enzymes perhaps exerts little adverse consequences on the human metabolism and biochemical network, thus rendering inhibitors oriented toward trehalose biosynthetic pathway an appealing potential target against fungal infections. ${ }^{44}$ Trehalose is regarded as an important alternative carbon source, providing energy for certain fungi. Trehalose-6-phosphate (T6P), a kind of trehalose intermediate, is crucial to the regulation of carbon metabolism, acting as a major signaling molecule in the fungal cell. ${ }^{45}$ In fungi, glycogen functions when nutrients are rich. However, with the depletion of glucose, trehalose begins to accumulate gradually and serves as an alternative energy source especially under stress and extreme starvation conditions. Trehalose can form hydrogen bonds by connecting two water molecules and thus functions as a substitute for water by interacting with the phospholipids or other macromolecules on the cell membrane so as to shield pathogenic fungi against stress. More interestingly, trehalose can also protect fungal cell membrane against dehydrated conditions by creating a glass state in cases where there is no water retention or crystallization. ${ }^{46,47}$

It is well acknowledged that reactive oxygen species (ROS) are crucial for the immune system to fight against pathogenic fungi. When confronted with oxidative stress conditions, trehalose acts as a free radical scavenger to eradicate ROS. ${ }^{48}$ Inhibition of trehalose biosynthesis in vivo may increase the effect of ROS-dependent host immune defense against fungi, as evidenced by the distinctly enhanced susceptibility of trehalose-deficient mutated $S$. cerevisiae to hydrogen peroxide $\left(\mathrm{H}_{2} \mathrm{O}_{2}\right)$ compared with the wild type. ${ }^{48}$ After being exposed to oxidative stress factors, reduced viability and poor survival outcomes were also observed in trehalose-deficient $C$. albicans, C. neoformans and $A$. fumigatus. ${ }^{49-51} \mathrm{~A}$ variety of trehalose-deficient mutant fungi including C. albicans, C. neoformans and Aspergillus nidulans are more sensitive to thermal stress, as suggested by a significant decrease in viability both in cold and in hot conditions..$^{50,52}$ Although there has been no strong evidence supporting the fact that trehalose constitutes an integral structural component of fungal cell wall, deficiency of Tps2 
in C. albicans, $A$. nidulans and A. fumigatus attenuates cell wall homeostasis and virulence by reducing the chitin and thus leads to cell lysis in high or low temperature. ${ }^{53}$ In $S$. cerevisiae, Tps $3 p$ is found to play a regulatory role in the Tps complex, especially during heat shock. Phosphorylated Tps3p also regulates the activity of Tps2. In C. albicans, a transcriptional factor called Cap $1 p$ is closely associated with tolerance to oxidative stress and the binding site for Cap $1 \mathrm{p}$ is located in the Tps 3 promoter region. Exposure to $\mathrm{H}_{2} \mathrm{O}_{2}$ results in a dramatic elevation in Tps3 mRNA. ${ }^{54}$ After elucidating the significance of trehalose synthesis in fungal survival and adaptation in response to various stresses, we conclude that Tps $1, T p s 2$ and their regulatory subunit Tps $3 p$ have the potential to be the ideal antifungal target.

Guirao-Abad et al have identified that validamycin A will be a promising substrate for designing new antifungal compounds targeting trehalose pathway in the future despite the fact that validamycin A cannot be used as a clinical antifungal agent at present. ${ }^{55}$ Magalhães et al have further confirmed that T6P inhibits Tps1 activity in $S$. cerevisiae, C. albicans and $C$. tropicalis, suggesting that T6P might be a promising antifungal candidate against pathogens the virulence of which depends on trehalose. However, as a phosphorylated molecule, T6P, is characterized by poor cell membrane permeability. So, routes of administration and drug delivery strategy must be explored to improve cell permeability. ${ }^{56}$

\section{Targeting sphingolipids}

Sphingolipids are important ingredients of fungal cell membrane. Some kinds of sphingolipids, such as inositolphosphoryl ceramide (IPC) and glucosylceramide (GlcCer), play critical roles in fungal pathogenesis and virulence. ${ }^{57} \mathrm{Up}$ till now, studies on the role of IPC in virulence are mainly restricted to non-virulent $S$. cerevisiae model ${ }^{58}$ C. neoformans strains which lack GlcCer synthase (Gcs1) gene not only have been found to lose their virulence in animal model but are also not influenced by other virulence factors, such as capsule and melanin. ${ }^{59}$ GlcCer may facilitate fungal tolerance to alkaline conditions as evidenced by an obvious growth retardness in an alkaline/neutral $\mathrm{pH}$ environment due to deletion of GlcCer synthase gene in C. neoformans. ${ }^{60}$ This is also the case with $C$. neoformans mutant lacking the sphingolipid methyltransferase 1 (Smt1), which yields unmethylated GlcCer. ${ }^{61}$ In C. albicans, disruption of genes which encode enzymes involved in GlcCer biosynthetic pathway, such as Smt1 and Gcs1, results in reduced cellular elongation in hyphal-inducing solid media. Knock-out of Gcs1 genes in C. albicans leads to incompleteness of cell membrane structure and enhanced susceptibility to fluconazole treatment. ${ }^{24,62}$

FEN1 and SUR4 are sphingolipid biosynthetic pathway genes that encode fatty acid elongases. Sharma et al have demonstrated that deletion of FEN1 and SUR4 in S. cerevisiae and their orthologs in C. albicans dramatically enhanced their sensitivity to AmB compared to their parent strains. This can be explained by the fact that depletion of sphingolipids may render ergosterol more easily accessible to AmB binding, thereby sensitizing cells to AmB ${ }^{63}$ Bari et al have found that overexpression of PMP3 gene, which encodes Pmp3 protein, culminates in elevated AmB resistance in both $S$. cerevisiae and $C$. albicans strains. While deletion of $P M P 3$ gene decreases their AmB resistance. Modulation of AmB resistance mediated by Pmp3 has nothing to do with ergosterol content, cell wall integrity and Hsp90, but is dependent on sphingolipid biosynthetic pathway. This can be manifested by the fact that AmB sensitivity of $P M P 3$ deletants can be suppressed by the addition of phytosphingosine, a sphingolipid pathway intermediate. ${ }^{64}$ As mentioned previously, biofilm, a complex consisting of yeast, pseudohyphae and hyphae, is usually surrounded by a protective layer called extracellular matrix to evade antifungals. Immunocompromised patients, especially those with long-term implanted medical devices, are more prone to Candida biofilm evolution. Alfatah et al have confirmed that genes CaFEN1 and CaFEN12 involved in sphingolipid biosynthesis are crucial for cell wall integrity and for the formation of hyphae and biofilms in C.albicans. $S$. cerevisiae deleted of FEN1 and SUR4 genes and C. albicans deleted of their orthologous genes (CaFEN1 and CaFEN12) are defective in sphingolipid biosynthesis, culminating in impaired cell wall integrity and disrupted formation of hyphae and biofilm. All these perhaps contribute to enhanced sensitivity of FEN1 and SUR4 deletants to AmB. ${ }^{65}$

There is great promise for the introduction of potential drugs specifically targeting fungal sphingolipids. These potential drugs can be divided into two broad categories: drugs that target biosynthesis of fungal sphingolipids and drugs that target the function of sphingolipids directly. The advantages and disadvantages of potential drugs are summarized in Table $1.58,66-76$

\section{Other new drugs Carboxymethyl chitosan (CM-chitosan)}

With the wide application of indwelling or implanted medical devices, there has been a drastic increase in Candida infection-related biofilm formation detected on the surfaces of these devices. ${ }^{77}$ Chitosan, a kind of $N$-deacetylated 
Table I Candidate drugs targeting sphingolipids

\begin{tabular}{|c|c|c|}
\hline Drugs & Advantages & Disadvantages \\
\hline \multicolumn{3}{|c|}{ Targeting the biosynthesis of fungal sphingolipids (inhibitors of sphingolipid-metabolizing enzymes) } \\
\hline $\begin{array}{l}\text { SPT inhibitors (myriocin, } \\
\text { sphingofungin) } \\
\text { Ceramide synthase inhibitors } \\
\text { (australifungin, fumonisin BI) } \\
\text { Inhibitors of SIP, sphingosine kinases } \\
\text { and SIP lyase } \\
\text { IPC inhibitors (aureobasidin, } \\
\text { galbonolide, khafrefungin, rustmicin) } \\
\text { GlcCer synthase inhibitors } \\
\text { (D-threo-PDMP) } \\
\text { GlcCer inhibitors (BHBM, D0) }\end{array}$ & $\begin{array}{l}\text { Impair biofilm formation; effective against Candida and } \\
\text { Aspergillus spp. } \\
\text { Have broad spectrum of antifungal effect against Candida and } \\
\text { Aspergillus spp. as well as Cryptococcus neoformans } \\
\text { Highly toxic to fungi; improve the host immune response } \\
\text { Active against Candida albicans and C. neoformans; impair } \\
\text { biofilm formation; Ipcl is not present in human cells } \\
\text { Have antifungal effect against Aspergillus fumigatus and } \\
\text { Aspergillus nidulans } \\
\text { Show antifungal activity against Candida spp., C. neoformans, } \\
\text { A. fumigatus and Histoplasma capsulatum in vitro and in vivo; } \\
\text { well tolerated in animal model }\end{array}$ & $\begin{array}{l}\text { Lack antifungal effect against mold, such as } \\
\text { Aspergillus spp. } \\
\text { Toxic to host; off-target effect; have no } \\
\text { significant effect against } C \text {. neoformans } \\
\text { Require further study }\end{array}$ \\
\hline \multicolumn{3}{|c|}{ Targeting the function of sphingolipids directly (targeting GlcCer directly) } \\
\hline RsAFP2 (defensins) & $\begin{array}{l}\text { Have no cross interactions with human GlcCer; active } \\
\text { against } C \text {. albicans; impair the transition from yeast to hyphae }\end{array}$ & $\begin{array}{l}\text { Strains lacking GlcCer synthase gene are } \\
\text { resistant to RsAFP2 (such as Candida } \\
\text { glabrata) }\end{array}$ \\
\hline Monoclonal antibodies against GlcCer & $\begin{array}{l}\text { Inhibit conidia germination; facilitate phagocytosis by } \\
\text { activating macrophages; present a synergistic effect with } \\
\text { other antifungals }\end{array}$ & Antifungal spectrum is relatively narrow \\
\hline
\end{tabular}

Abbreviations: BHBM, N'-(3-bromo-2-hydroxybenzylidene)-2-methylbenzohydrazide; D-threo-PDMP, D-threo-I-phenyl-2-decanoylamino-3-morpholino-I-propanol; D0, 3-bromo-N'-(3-bromo-4-hydroxybenzylidene) benzohydrazide; GlcCer, glucosylceramide; IPC, inositolphosphoryl ceramide; SIP, sphingosine-I-phosphate; SPT, serine palmitoyltransferase.

derivative of chitin, is characterized by the properties of being bioadhesive, biocompatible, biodegradable and nontoxic. Previous report has it that chitosan can inhibit hyphal growth and spore germination of various fungi. ${ }^{78}$ Several studies have reported strong anti-biofilm formation effect of chitosan and its derivatives on C. albicans. ${ }^{79-81}$ As can be observed through scanning electron microscopy, the biofilm was dramatically less dense on the silicone surface treated with CM-chitosan (a kind of chitosan derivative) compared to the control surface. This indicates that CM-chitosan can effectively prevent Candida spp. from adhering to surface of medicinal silicone rubber (such as voice prostheses) and inhibit the evolution of mixed fungi biofilm at different stages for a long period of time in vitro. ${ }^{82,83}$ Recently, Tan et al have revealed that $\mathrm{CM}$-chitosan has exerted a strong inhibitory effect on the biofilm formation of non-C. albicans species including C. tropicalis, Candida krusei, C. parapsilosis and C. glabrata, particularly on mixed-species biofilm. ${ }^{84}$ Yet, there is pressing need for further studies to explore whether medicinal silicone coated with CM-chitosan has an inhibitory effect on mixed fungal biofilm in vivo.

\section{Silver nanoparticles (AgNPs)}

AgNPs, the nanomaterials which are most commonly used to combat fungi, are characterized by high electrical conductivity, high thermal conductivity and high catalytical activity. AgNPs can be synthesized through environmentalfriendly approaches, one of which is chemical reduction with cysteine. ${ }^{85}$ AgNPs are more effective than AmB, posaconazole, itraconazole, voriconazole and caspofungine regarding in vitro antifungal activity. Artunduaga Bonilla et al confirmed that AgNPs were 640-fold more effective than fluconazole and particularly had antifungal effects against fluconazole-resistant Candida spp. They also reported the tiny cytotoxic effect of AgNPs on murine fibroblast cell lines. ${ }^{86}$ Besides, nanoparticles can interfere with superoxide dismutase, leading to accumulation of ROS, which stimulates inflammatory response and results in mitochondrial destruction. ${ }^{87}$

\section{Chromogranin A-N46 (CGA-N46)}

CGA is a kind of soluble protein which is predominantly expressed in neurons and endocrine cells. CGA-N46, a weak alkaline $\alpha$-helical peptide which corresponds to the N-terminal Pro31-Gln76 sequence (the 31st to 76th amino acids) of human CGA, has recently been identified and then produced in engineered Bacillus subtilis strain DB1342 by Li et al. ${ }^{88}$ Their team reported that at the relatively low concentration of $<0.8 \mathrm{mM}$, CGA-N46 demonstrated strong antifungal effect against all the tested Candida spp., including C. glabrata, $C$. parapsilosis, C. krusei, C. tropicalis and C. albicans. However, even at the high concentration of $3.2 \mathrm{mM}$, CGA-N46 
showed no inhibitory potency on the growth of filamentous fungi or other types of fungi, such as A. fumigatus, Aspergillus flavus, Aspergillus niger, C. neoformans, Fusarium moniliforme, some Microsporum spp. and Trichophyton spp. When combined with terbinafine and fluconazole, their antifungal effect against Candida spp., particularly against $C$. krusei, could be significantly augmented. Besides, their antifungal activity remained highly stable at $<40^{\circ} \mathrm{C}$ with a wide spectrum of $\mathrm{pH}$ (from $\mathrm{pH} 5.0$ to $\mathrm{pH} 7.0$ ). ${ }^{89} \mathrm{Li}$ et al also confirmed that CGA-N46 facilitated instability of phospholipid monolayer of cell membrane via disturbing the arrangement of phospholipids, thus leading to transportation of CGA-N46 into the cytoplasm without destroying the integrity or drilling pores in outer cell membrane of Candida spp. Treatment of C. krusei cells with CGA-N46 also resulted in severe damage of mitochondrial membrane, vacuolation of mitochondria, deformation or even degeneration of mitochondrial ridge and blockade of mitochondrial electron transfer chain by decreasing the potential of mitochondrial membrane, all of which led to cell death. The results of PCR confirmed that CGA-N46 inhibited DNA biosynthesis of Candida spp. in vitro and in vivo by inactivating DNA polymerase. ${ }^{90}$

Based on bioinformatic analysis, a variety of CGA-N46 derivatives was identified after removing some related amino acid sequences from CGA-N46. CGA-N8, CGA-N12, CGA-N15 and CGA-N16 were the final derived peptides synthesized by solid-phase peptide synthesis method and purified by HPLC. CGA-N12 was the inner sequence of CGA-N16, and CGA-N16 was located within CGA-N46. CGA-N12, CGA-N16 and CGA-N46 possessed the same active domain. CGA-N8 and CGA-N12 showed stronger stability than the other derivatives. All the derived peptides exhibited stronger anti-candidal effects than their "mother" peptide CGA-N46, among which CGA-N12 demonstrated the strongest antifungal activity and the weakest hemolytic toxicity, and hence was found to be a promising drug for the treatment of candidiasis..$^{91}$

\section{Conclusion and future perspectives}

Drug resistance is mushrooming at an alarming rate largely due to the limited categories of conventional antifungal drugs. A better understanding of drug resistance mechanisms illuminates new approaches to enhance therapeutic efficacy of current antifungal armamentarium and minimize host toxicity. The various underlying mechanisms of drug resistance have yet to be further elucidated. We have every reason to believe that targeting Hsp90/21, calcineurin, trehalose biosynthesis and sphingolipids biosynthesis and application of new-fashioned drugs, such as CM-chitosan, AgNPs, CGA-N46, CGA-N12, will definitely provide encouraging strategies to thwart fungal pathogens and thus form the rationale for the combination therapy with novel drugs in the treatment of refractory fungal infections. What is more, inhibition of biofilm formation, filamentation, drug efflux pump or mitochondrion also serves as a promising strategy to combat pathogenic fungi. ${ }^{92-95}$ However, some potential drugs are toxic. Therefore, therapeutic drug monitoring and careful surveillance should be conducted to determine drug exposure including dose, concentrations, formulations and routes of administration, with the intention of optimizing treatment response and minimizing side effects. Last, appropriate drug stewardship and patient management are also crucial in preventing drug resistance.

\section{Acknowledgments}

The authors extend their sincere appreciation to all the librarians who work in the library of Fourth Military Medical University for their generous assistance. The authors received no funding from any organization, institute or person.

\section{Disclosure}

The authors report no conflicts of interest in this work.

\section{References}

1. Brown GD, Denning DW, Gow NA, Levitz SM, Netea MG, White TC. Hidden killers: human fungal infections. Sci Transl Med. 2012; 4(165): 165 rv 13 .

2. Guarner J, Brandt ME. Histopathologic diagnosis of fungal infections in the 21st century. Clin Microbiol Rev. 2011;24(2):247-280.

3. Roemer T, Krysan DJ. Antifungal drug development: challenges, unmet clinical needs, and new approaches. Cold Spring Harb Perspect Med. 2014;4(5):a019703.

4. Esquivel BD, Smith AR, Zavrel M, White TC. Azole drug import into the pathogenic fungus Aspergillus fumigatus. Antimicrob Agents Chemother. 2015;59(6):3390-3398.

5. Perlin DS. Current perspectives on echinocandin class drugs. Future Microbiol. 2011;6(4):441-457.

6. Mohr J, Johnson M, Cooper T, Lewis JS, Ostrosky-Zeichner L. Current options in antifungal pharmacotherapy. Pharmacotherapy. 2008;28(5):614-645.

7. te Welscher YM, van Leeuwen MR, de Kruijff B, Dijksterhuis J, Breukink E. Polyene antibiotic that inhibits membrane transport proteins. Proc Natl Acad Sci U S A. 2012;109:11156-11159.

8. Li J, Buchner J. Structure, function and regulation of the hsp 90 machinery. Biomed J. 2013;36:106-117.

9. Leach MD, Klipp E, Cowen LE, Brown AJ. Fungal Hsp90: a biological transistor that tunes cellular outputs to thermal inputs. Nat Rev Microbiol. 2012;10(10):693-704.

10. Meyer P, Prodromou C, Hu B, et al. Structural and functional analysis of the middle segment of hsp90: implications for ATP hydrolysis and client protein and cochaperone interactions. Mol Cell. 2003;11(3): 647-658.

11. Taipale M, Jarosz DF, Lindquist S. HSP90 at the hub of protein homeostasis: emerging mechanistic insights. Nat Rev Mol Cell Biol. 2010; 11(7):515-528. 
12. Donlin LT, Andresen C, Just S, et al. Smyd2 controls cytoplasmic lysine methylation of Hsp90 and myofilament organization. Genes Dev. 2012;26(2):114-119.

13. Shapiro RS, Robbins N, Cowen LE. Regulatory circuitry governing fungal development, drug resistance, and disease. Microbiol Mol Biol Rev. 2011;75(2):213-267.

14. Singh SD, Robbins N, Zaas AK, Schell WA, Perfect JR, Cowen LE. Hsp90 governs echinocandin resistance in the pathogenic yeast Candida albicans via calcineurin. PLoS Pathog. 2009;5(7):e1000532.

15. Singh-Babak SD, Babak T, Diezmann S, et al. Global analysis of the evolution and mechanism of echinocandin resistance in Candida glabrata. PLoS Pathog. 2012;8(5):e1002718.

16. Cowen LE, Singh SD, Köhler JR, et al. Harnessing Hsp90 function as a powerful, broadly effective therapeutic strategy for fungal infectious disease. Proc Natl Acad Sci U S A. 2009;106(8):2818-2823.

17. Lamoth F, Juvvadi PR, Gehrke C, Steinbach WJ. In vitro activity of calcineurin and heat shock protein 90 Inhibitors against Aspergillus fumigatus azole- and echinocandin-resistant strains. Antimicrob Agents Chemother. 2013;57(2):1035-1039.

18. Lafayette SL, Collins $\mathrm{C}, \mathrm{Zaas} \mathrm{AK}$, et al. PKC signaling regulates drug resistance of the fungal pathogen Candida albicans via circuitry comprised of Mkc1, calcineurin, and Hsp90. PLoS Pathog. 2010;6(8): e1001069.

19. Shapiro RS, Zaas AK, Betancourt-Quiroz M, Perfect JR, Cowen LE. The Hsp90 co-chaperone Sgt1 governs Candida albicans morphogenesis and drug resistance. PLoS One. 2012;7(9):e44734.

20. Diezmann S, Michaut M, Shapiro RS, Bader GD, Cowen LE. Mapping the Hsp90 genetic interaction network in Candida albicans reveals environmental contingency and rewired circuitry. PLoS Genet. 2012; 8(3):e1002562.

21. Robbins N, Leach MD, Cowen LE. Lysine deacetylases Hda1 and Rpd3 regulate Hsp90 function thereby governing fungal drug resistance. Cell Rep. 2012;2(4):878-888.

22. Wu S, Wang Y, Liu N, Dong G, Sheng C. Tackling Fungal Resistance by Biofilm Inhibitors. J Med Chem. 2017;60(6): 2193-2211.

23. Robbins N, Uppuluri P, Nett J, et al. Hsp90 governs dispersion and drug resistance of fungal biofilms. PLoS Pathog. 2011;7(9):e1002257.

24. Noble SM, French S, Kohn LA, Chen V, Johnson AD. Systematic screens of a Candida albicans homozygous deletion library decouple morphogenetic switching and pathogenicity. Nat Genet. 2010;42(7):590-598.

25. Gow NAR, van de Veerdonk FL, Brown AJP, Netea MG. Candida albicans morphogenesis and host defence: discriminating invasion from colonization. Nat Rev Microbiol. 2012;10(2):112-122.

26. Shapiro RS, Sellam A, Tebbji F, Whiteway M, Nantel A, Cowen LE. Pho85, Pcl1, and Hms1 signaling governs Candida albicans morphogenesis induced by high temperature or Hsp90 compromise. Curr Biol. 2012;22(6):461-470.

27. Mayer FL, Wilson D, Jacobsen ID, et al. Small but crucial: the novel small heat shock protein Hsp21 mediates stress adaptation and virulence in Candida albicans. PLoS One. 2012;7(6):e38584.

28. Mayer FL, Wilson D, Hube B. Hsp21 potentiates antifungal drug tolerance in Candida albicans. PLoS One. 2013;8(3):e60417.

29. Lamoth F, Juvvadi PR, Soderblom EJ, Moseley MA, Steinbach WJ. Hsp70 and the Cochaperone StiA (Hop) Orchestrate Hsp90-Mediated Caspofungin Tolerance in Aspergillus fumigatus. Antimicrob Agents Chemother. 2015;59(8):4727-4733.

30. Juvvadi PR, Lamoth F, Steinbach WJ. Calcineurin as a multifunctional regulator: Unraveling novel functions in fungal stress responses, hyphal growth, drug resistance, and pathogenesis. Fungal Biol Rev. 2014; 28(2-3):56-69.

31. Juvvadi PR, Lee SC, Heitman J, Steinbach WJ. Calcineurin in fungal virulence and drug resistance: Prospects for harnessing targeted inhibition of calcineurin for an antifungal therapeutic approach. Virulence. 2017;8(2):186-197.

32. da Silva Ferreira ME, Heinekamp T, Härtl A, et al. Functional characterization of the Aspergillus fumigatus calcineurin. Fungal Genet Biol. 2007;44(3):219-230.
33. Chen Y-L, Lehman VN, Lewit Y, Averette AF, Heitman J. Calcineurin Governs Thermotolerance and Virulence of Cryptococcus gattii. G3 (Bethesda). 2013;3(3):527-539.

34. Chen YL, Brand A, Morrison EL, et al. Calcineurin controls drug tolerance, hyphal growth, and virulence in Candida dubliniensis. Eukaryot Cell. 2011;10(6):803-819.

35. Maesaki S, Marichal P, Hossain MA, Sanglard D, vanden Bossche H, Kohno S. Synergic effects of tactolimus and azole antifungal agents against azole-resistant Candida albican strains. J Antimicrob Chemother. 1998;42(6):747-753

36. Cordeiro RA, Macedo RB, Teixeira CE, et al. The calcineurin inhibitor cyclosporin A exhibits synergism with antifungals against Candida parapsilosis species complex. J Med Microbiol. 2014;63(Pt 7):936-944.

37. Dannaoui E, Schwarz P, Lortholary O. In vitro interactions between antifungals and immunosuppressive drugs against zygomycetes. Antimicrob Agents Chemother. 2009;53(8):3549-3551.

38. Thakur M, Revankar SG. In vitro interaction of caspofungin and immunosuppressives against agents of mucormycosis. J Antimicrob Chemother. 2011;66(10):2312-2314.

39. Lee SC, Li A, Calo S, Heitman J. Calcineurin plays key roles in the dimorphic transition and virulence of the human pathogenic zygomycete Mucor circinelloides. PLoS Pathog. 2013;9(9):e1003625.

40. Lewis RE, Ben-Ami R, Best L, Albert N, Walsh TJ, Kontoyiannis DP. Tacrolimus enhances the potency of posaconazole against Rhizopus oryzae in vitro and in an experimental model of mucormycosis. J Infect Dis. 2013;207(5):834-841.

41. Pinchai N, Perfect BZ, Juvvadi PR, et al. Aspergillus fumigatus calcipressin $\mathrm{CbpA}$ is involved in hyphal growth and calcium homeostasis. Eukaryot Cell. 2009;8(4):511-519.

42. Thevelein JM, Hohmann S. Trehalose synthase: guard to the gate of glycolysis in yeast? Trends Biochem Sci. 1995;20(1):3-10.

43. Elbein AD, Pan YT, Pastuszak I, Carroll D. New insights on trehalose: a multifunctional molecule. Glycobiology. 2003;13(4):17R-27R.

44. Perfect JR, Tenor JL, Miao Y, Brennan RG. Trehalose pathway as an antifungal target. Virulence. 2017;8(2):143-149.

45. Wilson RA, Jenkinson JM, Gibson RP, Littlechild JA, Wang ZY, Talbot NJ. Tps1 regulates the pentose phosphate pathway, nitrogen metabolism and fungal virulence. EMBO J. 2007;26(15):3673-3685.

46. Wiemken A. Trehalose in yeast, stress protectant rather than reserve carbohydrate. Antonie Van Leeuwenhoek. 1990;58(3):209-217.

47. Miao Y, Tenor JL, Toffaletti DL, et al. Structural and In Vivo Studies on Trehalose-6-Phosphate Synthase from Pathogenic Fungi Provide Insights into Its Catalytic Mechanism, Biological Necessity, and Potential for Novel Antifungal Drug Design. MBio. 2017;8(4):e00643-17.

48. Benaroudj N, Lee DH, Goldberg AL. Trehalose accumulation during cellular stress protects cells and cellular proteins from damage by oxygen radicals. J Biol Chem. 2001;276(26):24261-24267.

49. Zaragoza O, Gonzalez-Parraga P, Pedreno Y, Alvarez-Peral FJ, Arguelles JC. Trehalose accumulation induced during the oxidative stress response is independent of TPS1 mRNA levels in Candida albicans. Int Microbiol. 2003;6:121-125.

50. Petzold EW, Himmelreich U, Mylonakis E, et al. Characterization and regulation of the trehalose synthesis pathway and its importance in the pathogenicity of Cryptococcus neoformans. Infect Immun. 2006; 74(10):5877-5887.

51. Al-Bader N, Vanier G, Liu H, et al. Role of trehalose biosynthesis in Aspergillus fumigatus development, stress response, and virulence. Infect Immun. 2010;78(7):3007-3018.

52. Fillinger $\mathrm{S}$, Chaveroche $\mathrm{MK}$, van Dijck $\mathrm{P}$, et al. Trehalose is required for the acquisition of tolerance to a variety of stresses in the filamentous fungus Aspergillus nidulans. Microbiology. 2001;147(Pt 7):1851-1862.

53. Puttikamonkul S, Willger SD, Grahl N, et al. Trehalose 6-phosphate phosphatase is required for cell wall integrity and fungal virulence but not trehalose biosynthesis in the human fungal pathogen Aspergillus fumigatus. Mol Microbiol. 2010;131(1):911.

54. Cao Y, Wang Y, Dai B, et al. Trehalose is an important mediator of Cap1p oxidative stress response in Candida albicans. Biol Pharm Bull. 2008;31(3):421-425. 
55. Guirao-Abad JP, Sánchez-Fresneda R, Valentín E, Martínez-Esparza M, Argüelles JC. Analysis of validamycin as a potential antifungal compound against Candida albicans. Int Microbiol. 2013;16(4):217-225.

56. Magalhães RS, de Lima KC, de Almeida DS, de Mesquita JF, Eleutherio EC. Trehalose-6-Phosphate as a Potential Lead Candidate for the Development of Tps1 Inhibitors: Insights from the Trehalose Biosynthesis Pathway in Diverse Yeast Species. Appl Biochem Biotechnol. 2017;181(3):914-924.

57. Heung LJ, Luberto C, del Poeta M, del PM. Role of sphingolipids in microbial pathogenesis. Infect Immun. 2006;74(1):28-39.

58. Rollin-Pinheiro R, Singh A, Barreto-Bergter E, del Poeta M, del PM. Sphingolipids as targets for treatment of fungal infections. Future Med Chem. 2016;8(12):1469-1484.

59. Rittershaus PC, Kechichian TB, Allegood JC, et al. Glucosylceramide synthase is an essential regulator of pathogenicity of Cryptococcus neoformans. J Clin Invest. 2006;116(6):1651-1659.

60. Saito K, Takakuwa N, Ohnishi M, Oda Y. Presence of glucosylceramide in yeast and its relation to alkali tolerance of yeast. Appl Microbiol Biotechnol. 2006;71(4):515-521.

61. Singh A, Rella A, Schwacke J, Vacchi-Suzzi C, Luberto C, del Poeta M. Transmembrane transporter expression regulated by the glucosylceramide pathway in Cryptococcus neoformans. BMC Res Notes. 2015; $8(1): 681$.

62. Oura T, Kajiwara S. Candida albicans sphingolipid C9-methyltransferase is involved in hyphal elongation. Microbiology. 2010;156(4): 1234-1243.

63. Sharma S, Alfatah M, Bari VK, Rawal Y, Paul S, Ganesan K. Sphingolipid biosynthetic pathway genes FEN1 and SUR4 modulate amphotericin B resistance. Antimicrob Agents Chemother. 2014;58(4): 2409-2414.

64. Bari VK, Sharma S, Alfatah M, Mondal AK, Ganesan K. Plasma Membrane Proteolipid 3 Protein Modulates Amphotericin B Resistance through Sphingolipid Biosynthetic Pathway. Sci Rep. 2015;5(1):9685.

65. Alfatah M, Bari VK, Nahar AS, Bijlani S, Ganesan K. Critical role for CaFEN1 and CaFEN12 of Candida albicans in cell wall integrity and biofilm formation. Sci Rep. 2017;7(1):40281.

66. Cerantola V, Guillas I, Roubaty C, et al. Aureobasidin A arrests growth of yeast cells through both ceramide intoxication and deprivation of essential inositolphosphorylceramides. Mol Microbiol. 2009;71(6): $1523-1537$.

67. Mcquiston T, Luberto C, del Poeta M, del PM. Role of sphingosine-1phosphate (S1P) and S1P receptor 2 in the phagocytosis of Cryptococcus neoformans by alveolar macrophages. Microbiology. 2011;157(5): 1416-1427.

68. Thevissen K, de Mello Tavares P, Xu D, et al. The plant defensin RsAFP2 induces cell wall stress, septin mislocalization and accumulation of ceramides in Candida albicans. Mol Microbiol. 2012;84(1): 166-180.

69. Bigaud M, Guerini D, Billich A, Bassilana F, Brinkmann V. Second generation S1P pathway modulators: research strategies and clinical developments. Biochim Biophys Acta. 2014;1841(5):745-758.

70. Del Poeta M, Nimrichter L, Rodrigues ML, Luberto C. Synthesis and biological properties of fungal glucosylceramide. PLoS Pathog. 2014;10(1):e1003832.

71. Rollin-Pinheiro R, Liporagi-Lopes LC, de Meirelles JV, Souza LM, Barreto-Bergter E. Characterization of Scedosporium apiospermum glucosylceramides and their involvement in fungal development and macrophage functions. PLoS One. 2014;9(5):e98149.

72. Weiler S, Braendlin N, Beerli C, et al. Orally active 7-substituted (4-benzylphthalazin-1-yl)-2-methylpiperazin-1-yl]nicotinonitriles as active-site inhibitors of sphingosine 1-phosphate lyase for the treatment of multiple sclerosis. J Med Chem. 2014;57(12):5074-5084.

73. Farnoud AM, Bryan AM, Kechichian T, Luberto C, del Poeta M, del PM. The Granuloma Response Controlling Cryptococcosis in Mice Depends on the Sphingosine Kinase 1-Sphingosine 1-Phosphate Pathway. Infect Immun. 2015;83(7):2705-2713.
74. Perdoni F, Signorelli P, Cirasola D, et al. Antifungal activity of Myriocin on clinically relevant Aspergillus fumigatus strains producing biofilm. BMC Microbiol. 2015;15(1):248.

75. Proia RL, Hla T. Emerging biology of sphingosine-1-phosphate: its role in pathogenesis and therapy. J Clin Invest. 2015;125(4): 1379-1387.

76. Candido K, Soufi H, Bandyopadhyay M, Dasgupta S. Therapeutic Impact of Sphingosine 1-phosphate Receptor Signaling in Multiple Sclerosis. Mini Rev Med Chem. 2016;16(7):547-554.

77. Leonhard M, Schneider-Stickler B. Voice prostheses, microbial colonization and biofilm formation. Adv Exp Med Biol. 2015;830:123-136.

78. Palma-Guerrero J, Jansson HB, Salinas J, Lopez-Llorca LV. Effect of chitosan on hyphal growth and spore germination of plant pathogenic and biocontrol fungi. J Appl Microbiol. 2008;104(2):541-553.

79. Costa E, Silva S, Tavaria F, Pintado M. Antimicrobial and Antibiofilm Activity of Chitosan on the Oral Pathogen Candida albicans. Pathogens. 2014;3(4):908-919.

80. $\mathrm{Pu}$ Y, Liu A, Zheng Y, Ye B, Ye B. In vitro damage of Candida albicans biofilms by chitosan. Exp Ther Med. 2014;8(3):929-934.

81. Silva-Dias A, Palmeira-de-Oliveira A, Miranda IM, et al. Anti-biofilm activity of low-molecular weight chitosan hydrogel against Candida species. Med Microbiol Immunol. 2014;203(1):25-33.

82. Tan Y, Leonhard M, Moser D, Ma S, Schneider-Stickler B. Long-term antibiofilm activity of carboxymethyl chitosan on mixed biofilm on silicone. Laryngoscope. 2016;126(12):E404-E408.

83. Tan Y, Leonhard M, Moser D, Ma S, Schneider-Stickler B. Inhibition of mixed fungal and bacterial biofilms on silicone by carboxymethyl chitosan. Colloids Surf B Biointerfaces. 2016;148:193-199.

84. Tan Y, Leonhard M, Moser D, Schneider-Stickler B. Antibiofilm activity of carboxymethyl chitosan on the biofilms of non-Candida albicans Candida species. Carbohydr Polym. 2016;149:77-82.

85. Sukhwal A, Jain D, Joshi A, Rawal P, Kushwaha HS. Biosynthesised silver nanoparticles using aqueous leaf extract of Tagetes patula L. and evaluation of their antifungal activity against phytopathogenic fungi. IET Nanobiotechnol. 2017;11(5):531-537.

86. Artunduaga Bonilla JJ, Paredes Guerrero DJ, Sánchez Suárez CI, Ortiz López CC, Torres Sáez RG. In vitro antifungal activity of silver nanoparticles against fluconazole-resistant Candida species. World $J$ Microbiol Biotechnol. 2015;31(11):1801-1809.

87. StensbergMC,WeiQ,MclamoreES, PorterfieldDM, WeiA, SepúlvedaMS. Toxicological studies on silver nanoparticles: challenges and opportunities in assessment, monitoring and imaging. Nanomedicine. 2011; 6(5):879-898.

88. Li RF, Wang B, Liu S, et al. Optimization of the Expression Conditions of CGA-N46 in Bacillus subtilis DB1342(p-3N46) by Response Surface Methodology. Interdiscip Sci. 2016;8(3):277-283.

89. Li RF, Lu YL, Lu YB, et al. Antiproliferative effect and characterization of a novel antifungal peptide derived from human Chromogranin A. Exp Ther Med. 2015;10(6):2289-2294.

90. Li RF, Yan XH, Lu YB, et al. Anti-candidal activity of a novel peptide derived from human chromogranin $\mathrm{A}$ and its mechanism of action against Candida krusei. Exp Ther Med. 2015;10(5):1768-1776.

91. Li RF, Lu ZF, Sun YN, et al. Molecular Design, Structural Analysis and Antifungal Activity of Derivatives of Peptide CGA-N46. Interdiscip Sci. 2016;8(3):319-326.

92. Desai JV, Mitchell AP, Andes DR. Fungal biofilms, drug resistance, and recurrent infection. Cold Spring Harb Perspect Med. 2014;4(10): a019729.

93. Holmes AR, Cardno TS, Strouse JJ, et al. Targeting efflux pumps to overcome antifungal drug resistance. Future Med Chem. 2016;8(12): 1485-1501.

94. Li D, Calderone R. Exploiting mitochondria as targets for the development of new antifungals. Virulence. 2017;8(2):159-168.

95. Vila T, Romo JA, Pierce CG, Mchardy SF, Saville SP, Lopez-Ribot JL. Targeting Candida albicans filamentation for antifungal drug development. Virulence. 2017;8(2):150-158. 


\section{Publish your work in this journal}

Drug Design, Development and Therapy is an international, peerreviewed open-access journal that spans the spectrum of drug design and development through to clinical applications. Clinical outcomes, patient safety, and programs for the development and effective, safe, and sustained use of medicines are the features of the journal, which

has also been accepted for indexing on PubMed Central. The manuscript management system is completely online and includes a very quick and fair peer-review system, which is all easy to use. Visit http://www.dovepress.com/testimonials.php to read real quotes from published authors.

Submit your manuscript here: http://www.dovepress.com/drug-design-development-and-therapy-journal 